\title{
Searches for heavy resonances decaying to pairs of massive vector bosons at CMS
}

\author{
A. Hinzmann* \\ On behalf of the CMS Collaboration \\ CERN, Switzerland \\ E-mail: hinzmannecern.ch
}

\begin{abstract}
Recent results of searches for heavy resonances decaying to pairs of Standard Model W and Z bosons at CMS using $8 \mathrm{TeV}$ LHC data are presented. Several new physics scenarios predict the existence of resonances that decay with high branching ratio to pairs of bosons, including extra dimensions and composite Higgs models. The final states considered here are WW, WZ, and $\mathrm{ZZ}$. The acceptance for $\mathrm{TeV}$ resonances is increased by applying jet substructure techniques to reconstruct $\mathrm{W}$ or $\mathrm{Z}$ bosons with high transverse momenta whose decay products merge into a single jet.
\end{abstract}

The European Physical Society Conference on High Energy Physics -EPS-HEP2013

18-24 July 2013

Stockholm, Sweden

\footnotetext{
* Speaker.
} 


\section{Introduction}

Many extensions of the Standard Model (SM) predict new resonances decaying to pairs of vector bosons. Therefore, CMS [1] has a wide program of searches for such resonances, considering the following benchmark models (references given in the cited analyses). 1. Randall-Sundrum gravitons $\mathrm{G}_{\mathrm{RS}} \rightarrow \mathrm{WW} / \mathrm{ZZ}$ where both the original model "RS1" as well as the "Bulk" graviton model with enhanced coupling of the $\mathrm{G}_{\mathrm{RS}}$ to WW/ZZ are considered. 2. Heavy partners of the SM W boson W' $\rightarrow$ WZ with couplings from the extended gauge model. 3. Low-scale technicolor (LSTC) models with $\rho_{\mathrm{TC}} \rightarrow$ WZ. 4. SM Higgs-like boson $\mathrm{H} \rightarrow$ WW/ZZ in addition to the SM Higgs boson at $125 \mathrm{GeV}$.

Table 1 summarizes the CMS searches for vector boson pairs categorized by final states and resonance mass range. The low resonance mass range around $125 \mathrm{GeV}$ is covered by the SM Higgs boson searches which are not detailed in this contribution. The high resonance mass searches are categorized in searches in "boosted topologies" with high transverse momentum $\mathrm{W}$ and $\mathrm{Z}$ bosons whose hadronic decay products merge into a single jet or whose leptonic decay products have overlapping isolation cones, and non-boosted topologies covering intermediate resonance masses. The focus of this contribution is on the most recent searches which fall in the category of boosted topologies.

\begin{tabular}{|c|c|c|}
\hline Final state & non-boosted topologies & boosted topologies \\
\hline $\mathrm{ZZ} \rightarrow 1111$ & $\mathrm{H} \rightarrow \mathrm{ZZ}(7+8 \mathrm{TeV})[2]$ & \\
\hline $\mathrm{WZ} \rightarrow 111 v$ & & $\mathbf{W}^{\prime} / \rho_{\mathrm{TC}} \rightarrow \mathbf{W Z}(8 \mathrm{TeV})[3]$ \\
\hline $\mathrm{WW} / \mathrm{ZZ} \rightarrow 11 v v$ & $\begin{array}{l}\mathrm{H} \rightarrow \mathrm{WW}(7+8 \mathrm{TeV})[2] \\
\mathrm{H} \rightarrow \mathrm{ZZ}(8 \mathrm{TeV})\end{array}$ & \\
\hline $\mathrm{ZZ/WZ} \rightarrow \mathrm{llqq}$ & $\begin{array}{l}\mathrm{H} \rightarrow \mathrm{ZZ}(7+8 \mathrm{TeV})[2] \\
\mathrm{G}_{\mathrm{RS}} \rightarrow \mathrm{ZZ}(7 \mathrm{TeV})[5]\end{array}$ & $\mathrm{G}_{\mathrm{RS}} \rightarrow \mathrm{ZZ}, \mathrm{W}^{\prime} \rightarrow \mathrm{WZ}(7 \mathrm{TeV})[6]$ \\
\hline $\mathrm{WW} \rightarrow \mathrm{lvqq}$ & $\mathrm{H} \rightarrow \mathrm{WW}(7+8 \mathrm{TeV})[2]$ & $\mathbf{H} \rightarrow \mathbf{W W}(\mathbf{8} \mathrm{TeV})[7]$ \\
\hline $\mathrm{ZZ} \rightarrow v v \mathrm{qq}$ & & $\mathrm{G}_{\mathrm{RS}} \rightarrow \mathrm{ZZ}(7 \mathrm{TeV})[6]$ \\
\hline $\mathrm{WW} / \mathrm{WZ} / \mathrm{ZZ} \rightarrow \mathrm{qqqq}$ & & $\mathbf{G}_{\mathrm{RS}} \rightarrow \mathbf{W W} / \mathbf{Z Z}, \mathbf{W}^{\prime} \rightarrow \mathbf{W Z}(\mathbf{8} \mathrm{TeV})[8]$ \\
\hline
\end{tabular}

Table 1: Summary of searches for vector boson pairs in CMS. Analyses covered in this contribution are printed in bold face.

\section{2. $\mathbf{W}^{\prime} / \rho_{\mathrm{TC}} \rightarrow \mathbf{W Z} \rightarrow \mathbf{3 1}+\mathbf{E}_{\mathrm{T}}^{\mathrm{miss}}$}

In this search the $\mathrm{WZ}$ resonance mass $\mathrm{M}_{\mathrm{WZ}}$ is reconstructed from two opposite-sign sameflavor leptons falling in the $\mathrm{Z}$ mass window and one lepton plus $\mathrm{E}_{\mathrm{T}}^{\text {miss }}$ using the $\mathrm{W}$ mass constraint to estimate the neutrino momentum along the beam axis. Special identification and isolation requirements for leptons are used to enhance the efficiency for leptons from the high $\mathrm{p}_{\mathrm{T}} \mathrm{Z}$ bosons whose decay products have a small angular separation in the detector. One of the two muons from the $\mathrm{Z}$ is reconstructed with relaxed muon system requirements "tracker muons" and particle based lepton isolation is calculated excluding the other lepton from the Z. Figure 1 (top left) shows the reconstructed $\mathrm{M}_{\mathrm{WZ}}$ spectrum. The main background is $\mathrm{SM} \mathrm{WZ}$ production which is well modeled using MC simulation. 
An excess on top of the SM background is searched for using W' $\rightarrow$ WZ as a benchmark model. A counting experiment is performed in windows of $\mathrm{M}_{\mathrm{WZ}}$ and $\mathrm{L}_{\mathrm{T}}=\sum \mathrm{p}_{\mathrm{T}}^{\text {lep }}$ optimized for each $\mathrm{W}$ ' mass separately as demonstrated in Fig. 1 (top right). Figure 1 (bottom left) shows the resulting cross section limit. No significant excess is observed. W' $\rightarrow$ WZ is therefore excluded in the mass range 0.17 to $1.45 \mathrm{TeV}$. In Fig. 1 (bottom right) the search is interpreted in terms of the LSTC model where the limit depends on the $\pi_{\mathrm{TC}}$ mass since the $\rho_{\mathrm{TC}}$ can decay to $\mathrm{WZ}$ and to $\mathrm{W} \pi_{\mathrm{TC}}$. The LSTC limits are the most stringent to date.
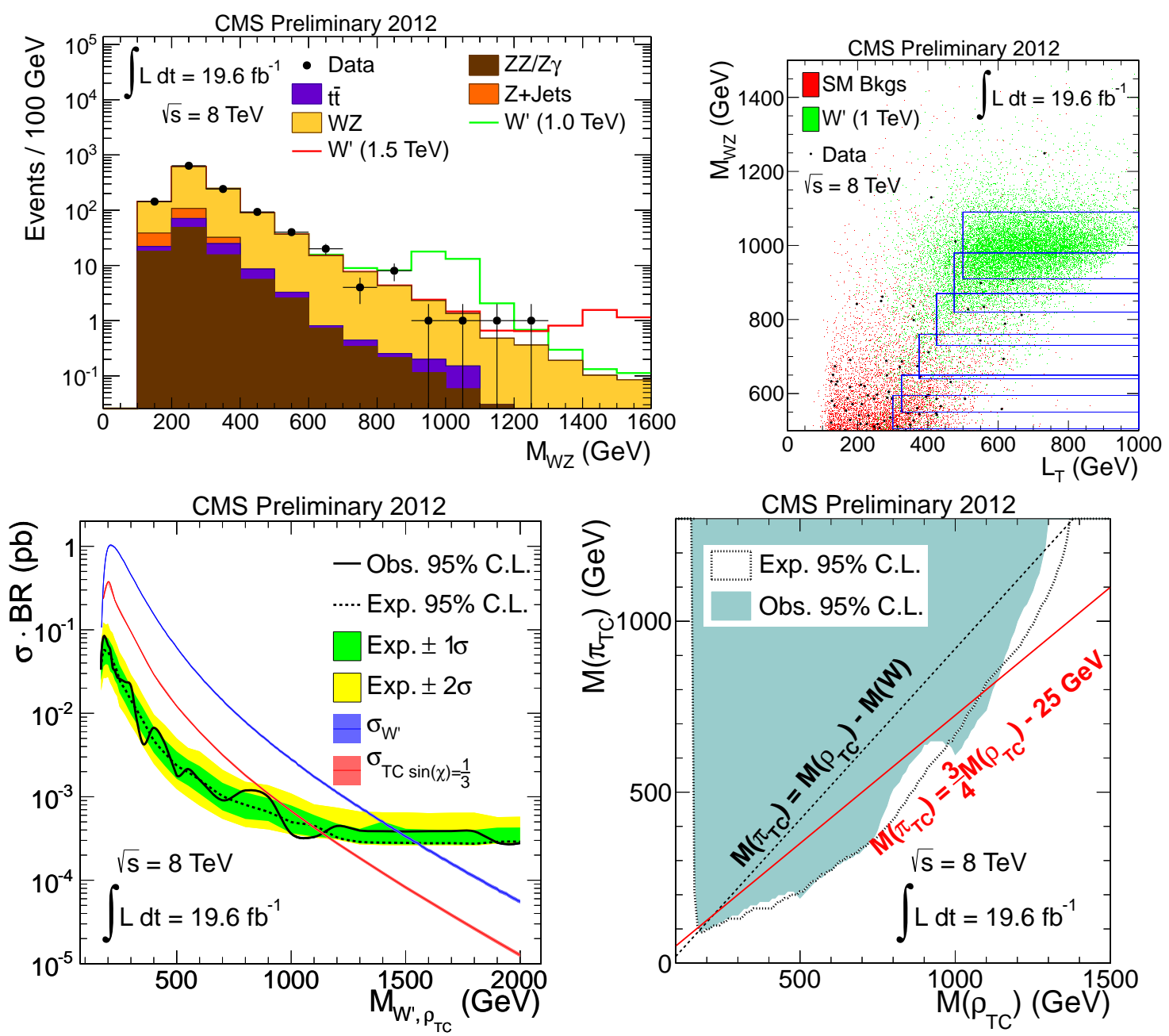

Figure 1: Summary of the $\mathrm{W}^{\prime} / \rho_{\mathrm{TC}} \rightarrow \mathrm{WZ} \rightarrow 31+\mathrm{E}_{\mathrm{T}}^{\text {miss }}$ search.

\section{3. $\mathrm{H} \rightarrow \mathrm{WW} \rightarrow \mathbf{1 l}+\mathbf{1}$ jet $+\mathbf{E}_{\mathrm{T}}^{\text {miss }}$}

In this search the WW resonance mass $\mathrm{m}_{1 v \mathrm{j}}$ in Fig. 2 (top left) is reconstructed from one jet with a size $\mathrm{R}=0.8$ to reconstructed a $\mathrm{W}$ whose hadronic decay products merge into a single jet and one lepton plus $\mathrm{E}_{\mathrm{T}}^{\mathrm{miss}}$ using the $\mathrm{W}$ mass constraint to estimate the neutrino momentum along the beam axis. Jet substructure techniques are used to reject backgrounds with quark and gluon as opposed to $\mathrm{W}$ initiated jets. The "pruned" jet mass where soft and large angle particles are 
rejected from the jet clustering and the $\mathrm{N}$-subjettiness ratio $\tau_{2} / \tau_{1}$ are used for discrimination. $\mathrm{A}$ semileptonic $t \bar{t}$ data sample containing high $\mathrm{p}_{\mathrm{T}} \mathrm{W}$ bosons is used to derive data-MC scale factors and estimate systematic uncertainties for both discriminators as demonstrated in Fig. 2 (top right). The main background from $\mathrm{W}+$ jets events is estimated from the pruned jet mass side-band in data shown in Fig. 2 (bottom left), extrapolated to the W mass window using MC simulations. This channel has also a non-vanishing background from SM WW production visible in the same figure.

No significant excess is observed. Therefore limits are set in the context of a beyond SM heavy singlet scalar additional to the Higgs boson at $125 \mathrm{GeV}$ where $\mathrm{C}^{\prime}$ is the scale factor for its coupling as shown in Fig. 2 (bottom right). Coupling measurements of the Higgs boson at $125 \mathrm{GeV}$ constrain the coupling $C^{\prime}$ to small values.
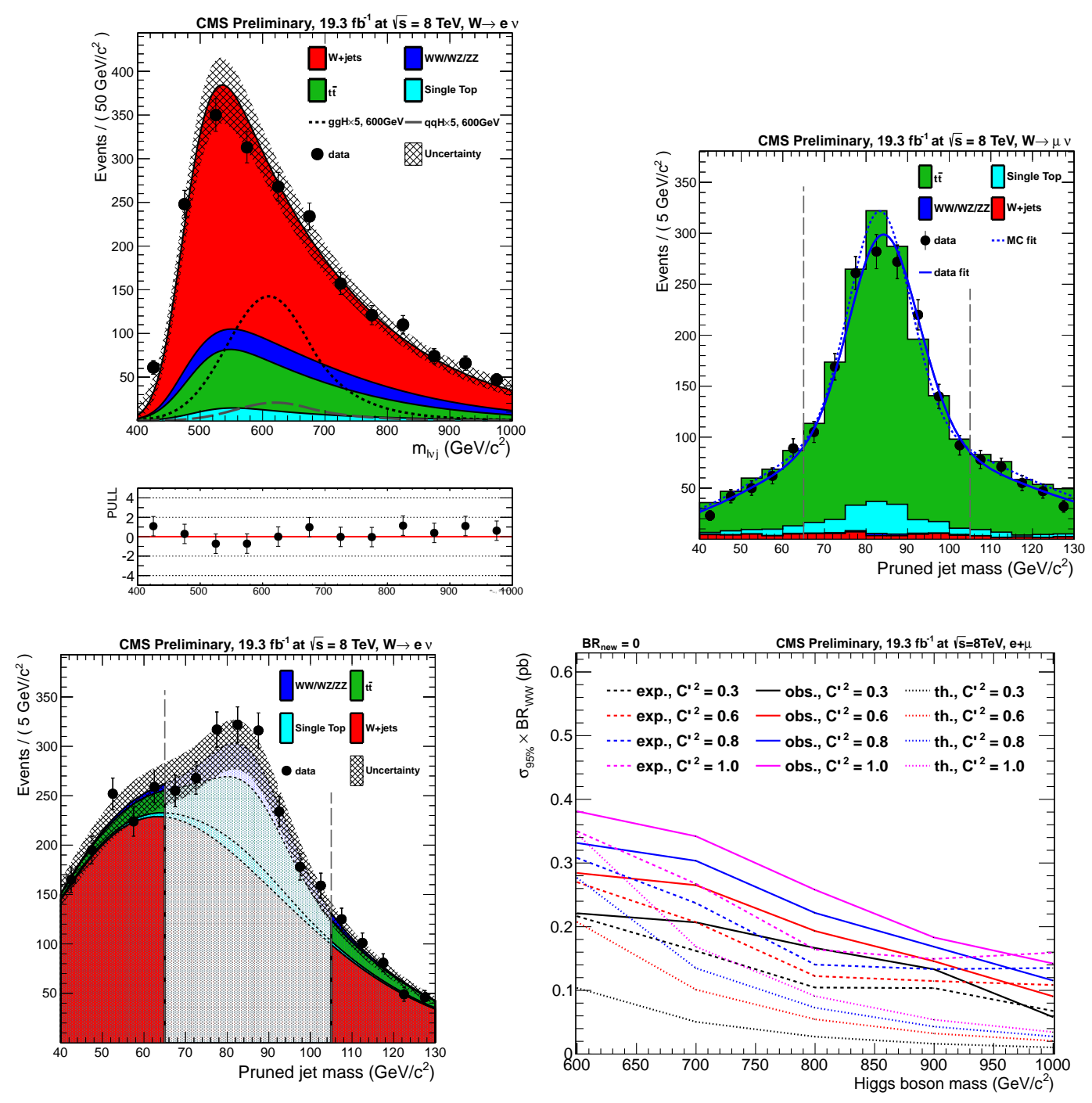

Figure 2: Summary of the $\mathrm{H} \rightarrow \mathrm{WW} \rightarrow 11+1$ jet $+\mathrm{E}_{\mathrm{T}}^{\text {miss }}$ search. 


\section{4. $\mathbf{G}_{\mathrm{RS}} \rightarrow \mathrm{WW} / \mathrm{ZZ}, \mathrm{W}^{\prime} \rightarrow \mathbf{W Z} \rightarrow \mathbf{2 j e t s}$}

In this search the WW/ZZ/WZ resonance mass is reconstructed from dijet events with a jet cone size of $\mathrm{R}=0.8$. The large $\mathrm{QCD}$ background is rejected by a cut on the angular distribution of the dijets $\left|\eta_{1}-\eta_{2}\right|<1.3$ and by W/Z-jet-tagging based on the pruned jet mass and the N-subjettiness ratio $\tau_{2} / \tau_{1}$. Since this search is sensitive to both jets from $\mathrm{W}$ and $\mathrm{Z}$ bosons as demonstrated in Fig. 3 (top left), the pruned jet mass window cut is chosen to be 70 to $100 \mathrm{GeV}$. The efficiency and background rejection of W/Z-tagging both jets is shown in Fig. 3 (top right). The background is estimated from a smooth fit (signal+background) to the data as shown in Fig. 3 (bottom left).

No significant excess is found and limits are set on various models. $G_{R S}\left(k / M_{P L}=0.1\right) \rightarrow W W(Z Z)$ is excluded in the mass range 1.0 to $1.59(1.17) \mathrm{TeV}$ (Fig. 3 (bottom right)) and $\mathrm{W}^{\prime} \rightarrow \mathrm{WZ}$ is excluded in the mass range 1.0 to $1.73 \mathrm{TeV}$, which are the most stringent limits to date on these channels. In addition, exclusion limits are also set on the $\mathrm{q}^{*} \rightarrow \mathrm{qW}(\mathrm{qZ})$ models where only one jet is required to be W/Z-tagged.
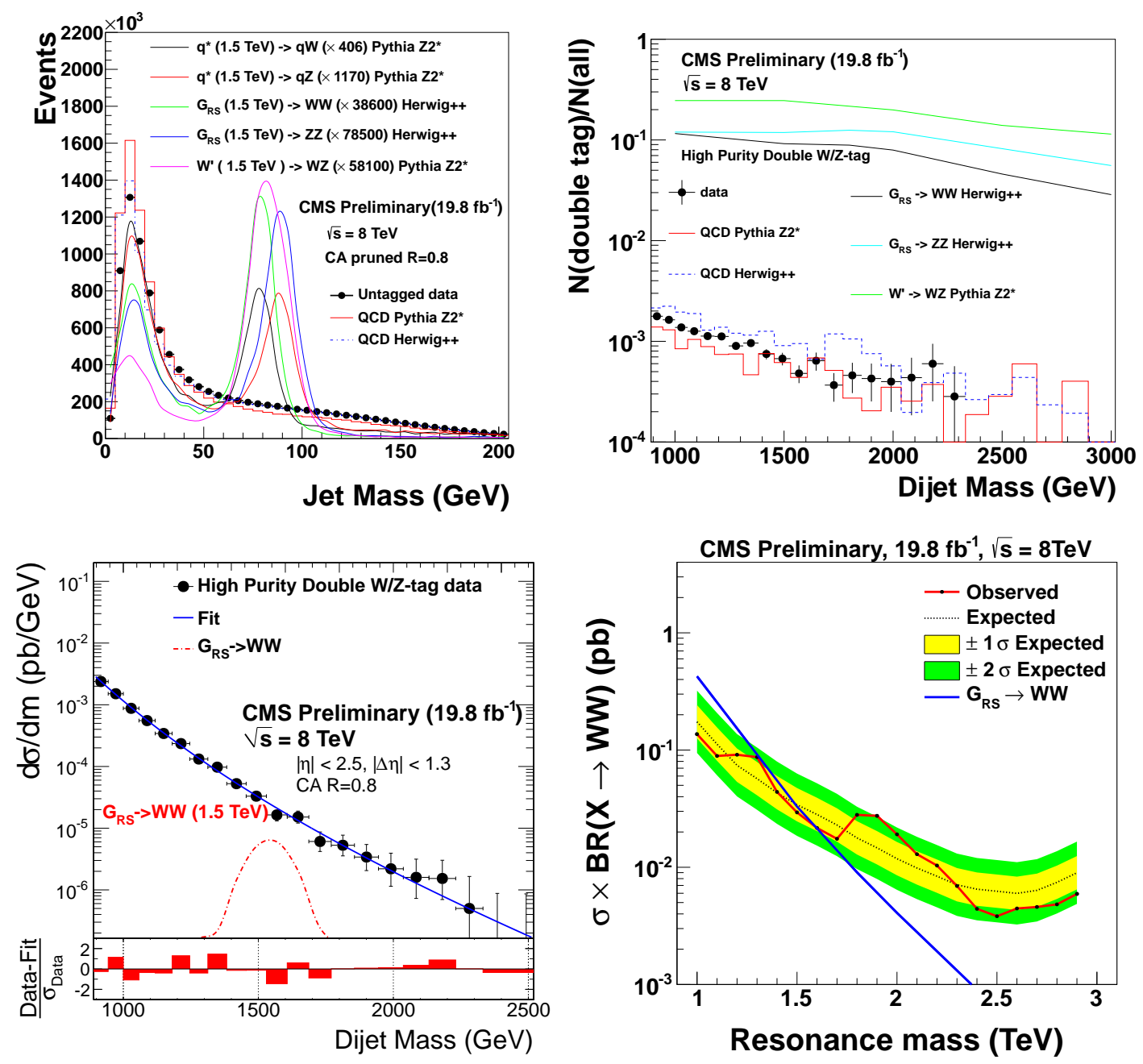

Figure 3: Summary of the $\mathrm{G}_{\mathrm{RS}} \rightarrow \mathrm{WW} / \mathrm{ZZ}, \mathrm{W}^{\prime} \rightarrow \mathrm{WZ} \rightarrow 2$ jets search. 


\section{Summary}

CMS has a broad program of searches for $\mathrm{VV}$ resonances aiming at covering all possible final states for best sensitivity over the full resonance mass range. A combination of all $8 \mathrm{TeV}$ searches is foreseen. Depending on the level of difficulty to model the background, various modeling techniques, based either on MC simulation, or on sidebands in the data, or on a smooth fit function, are applied. The analyses presented here set the most stringent limits on $\mathrm{G}_{\mathrm{RS}} \rightarrow \mathrm{WW} / \mathrm{ZZ}$ and W' $\rightarrow \mathrm{WZ}$. No discovery has been made so far in these final states, but since they occur in several extensions to the SM, searches in these channels remain highly interesting in the future of LHC data taking.

\section{References}

[1] CMS Collaboration, The CMS experiment at the CERN LHC, JINST 0803:S08004, 2008

[2] CMS Collaboration, Search for a standard-model-like Higgs boson with a mass in the range 145 to $1000 \mathrm{GeV}$ at the LHC, Eur. Phys. J. C 73 (2013) 2469, 10.1140/epjc/s10052-013-2469-8

[3] CMS Collaboration, Search for W'/technirho in WZ using leptonic final states, CMS-PAS-EXO-12-025 (2013) http://cds.cern.ch/record/1558197

[4] CMS Collaboration, Search for a heavy Higgs boson in the H to ZZ to 212nu channel in pp collisions at sqrt(s)= 7 and $8 \mathrm{TeV}$, CMS-PAS-HIG-13-014 (2013), http://cds.cern.ch/record/1546776

[5] CMS Collaboration, Search for a narrow, spin-2 resonance decaying to a pair of $Z$ bosons in the $q$ q-bar l+l-final state, Phys. Lett. B 718 (2013) 1208, 10.1016/j.physletb.2012.11.063

[6] CMS Collaboration, Search for exotic resonances decaying into WZ/ZZ in pp collisions at sqrt( $s)=7$ $\mathrm{TeV}$, JHEP 02 (2013) 036, 10.1007/JHEP02(2013)036

[7] CMS Collaboration, Search for a Standard Model-like Higgs boson decaying into WW to l nu qqbar in pp collisions at sqrt $s=8 \mathrm{TeV}$, CMS-PAS-HIG-13-008 (2013), http://cds.cern.ch/record/1546778

[8] CMS Collaboration, Search for heavy resonances in the W/Z-tagged dijet mass spectrum in pp collisions at $8 \mathrm{TeV}$, CMS-PAS-EXO-12-024 (2013), http://cds.cern.ch/record/1563153 